AUTORES:

Luan Gabriel de Souza

Suzy Silva Pinto

Romina K. da Silva Michiles

Kathya Augusta T. Lopes

Minerva L. de C. Amorim

Lionela da Silva Corrêa ${ }^{1}$

Universidade Federal do Amazonas - UFAM

\section{Avaliação da aptidão física \\ relacionada à saúde \\ de adolescentes e adultos com deficiência intelectual}

\section{PALAVRAS CHAVE:}

Aptidão física. Saúde. Adolescentes. Adultos.
Evaluation of physical fitness related

to the health of adolescents and adults

with intellectual disabilities

\section{ABSTRACT}

The aim of the study was to evaluate the health -related physical fitness of individuals 17 to 36 years, with mental deficiency Motor Activities Program for Persons with Disabilities (PROAMDE). The research is characterized as descriptive quantitative approach with 3 males and 3 females, characterized as a convenience sample. Data were collected through the following variables: arm flexion test (protocol CSTF, cited in Morrow et al, 2003), Abdominal endurance test of 1 minute and the horizontal thrust test. Data were analyzed and discussed from the literature although there are many studies in the area of health-related fitness assessment of people with intellectual disabilities in physical fitness variables. In general, the tests applied are classified as: excellent, good, average $(+)$, medium, medium (-), weak and very weak. The results indicated that the tested individuals are being ranked below average with a mean (-), weak and very weak. We conclude that the study of this group has assessed physical fitness levels below the desirable levels of fitness and there is need for other related physical fitness of individuals with intellectual disability studies.

KEY WORDS:

Physical fitness. Health. Teens. Adults. 


\section{INTRODUÇÃo}

O ser humano tem tido um aumento significativo no seu tempo de vida, proveniente de seus cuidados com a saúde, para ter um longo prazo de vida, deve-se considerar os níveis dignos de sobrevivência e de direitos humanos, em que o cidadão deve ter acesso aos avanços científicos e tecnológicos das diferentes áreas relacionadas à saúde ${ }^{1}$. Dessa forma, a atividade física torna-se um instrumento fundamental para o ser humano manter-se com bons níveis de aptidão física e boa qualidade de vida.

A aptidão física é um conjunto de atributos associados à capacidade de realizar atividade física em relação à composição genética do indivíduo, bem como a manutenção de uma nutrição adequada ${ }^{2}$.

Aptidão física refere-se ao aspecto biopsicossocial do ser humano, afirmando que o indivíduo estar apto para executar todas as suas atividades biológicas, psicológicas e sociais, the proporcionando uma integração adequada no meio em que ele está incluído ${ }^{3}$.

Aptidão física também pode ser definida como à capacidade funcional direcionada à execução de esforços físicos relacionados à prática de atividade física, representada por conjunto de componentes associados à saúde e ao desempenho atlético ${ }^{4}$

A aptidão associada à saúde é um estado relativo do ser, não necessariamente uma aptidão, habilidade ou capacidade, e influencia a melhoria da qualidade de vida e ao baixo índice de risco de doenças. A aptidão devidamente associada à performance é relativamente estável e está intimamente relacionada à habilidade atlética ${ }^{2}$

É indiscutível que a atividade física proporciona inúmeros benefícios psicomotores para pessoas com ou sem deficiência, deficiência pode ser definida como a perda ou anormalidade de estrutura ou função psicológica, fisiológica ou anatômica, de forma temporária ou permanente ${ }^{5}$. Acrescenta-se nestas a ocorrência de uma anomalia, a perda ou defeito de um membro, órgão, tecido ou qualquer outra estrutura do corpo, inclusive das funções mentais. Representa a exteriorização de um estado patológico, refletindo um distúrbio orgânico, uma perturbação no órgão. Dessa forma, o objetivo do trabalho foi avaliar a aptidão física de pessoas com deficiência intelectual, tendo em vista, oportunizar praticas de atividade física que condizem com a realidade encontrada do indivíduo, no intuito de ter um melhor cuidado com a saúde, condição física para as atividades diárias, diminuindo assim o risco de doenças.

\section{MATERIAIS E MÉTODO}

A pesquisa é caracterizada como descritiva com abordagem quantitativa, este tipo de pesquisa se constitui pelo objetivo de descrever as características de uma determinada população ou fenômeno, ou estabelecer relações entre variáveis, inclui-se também nesta pesquisa o objetivo de estudar características de um grupo em relação à: idade, sexo, procedência, nível de escolaridade, estado de saúde física e mental ${ }^{6}$

SUJEITOS DA PESQUISA

Por se tratar de um projeto piloto em que tínhamos como foco averiguar os benefícios do atletismo e suas influencias na vida das pessoas com deficiência, criamos um projeto de atletismo que surgiu da necessidade de oferecer pratica satisfatória de uma modalidade em que beneficiasse tanto o desempenho motor quanto a qualidade de vida do aluno. Optamos pelo desporto por ele servir de base para outras modalidades esportivas, além de envolver uma série de movimentos básicos e naturais. 0 atletismo é nomeado um esporte de base, pois sua prática influencia nos movimentos primordiais do ser humano ${ }^{7}$.

Para isso, selecionamos 6 indivíduos do Programa de Atividades Motoras Para Deficientes (PROAMDE) da Universidade Federal do Amazonas (UFAM), do sexo masculino e feminino, sendo 3 mulheres e 3 homens, com idade entre 17 a 36 anos.

CRITÉRIOS DE INCLUSÃO E EXCLUSÃO (ELEGIBILIDADE)

Os critérios utilizados para incluir os indivíduos foram: Adolescentes e adultos de 17 a 36 anos com deficiência intelectual, do gênero masculino e feminino autorizados pelos seus responsáveis. Os critérios de exclusão dos indivíduos foram: Alunos que não compareceram à coleta de dados e alunos que o responsável não autorizou para a aplicação dos testes.

INSTRUMENTOS DA PESQUISA

Os instrumentos utilizados na pesquisa foram: teste de resistência abdominal de 1 minuto (YMCA) que consiste em executar o maior número de repetições no tempo máximo de 1 minuto. 0 teste de resistência abdominal é um tanto controverso, pois tal execução não é utilizada apenas com os músculos abdominais, mas também com os flexores do quadril. Mesmo assim, o teste é muito utilizado ${ }^{8}$.

$\mathrm{Na}$ aplicação do teste, o avaliado deve se deitar em posição de decúbito dorsal em um colchonete com os joelhos flexionados e pés apoiados no solo. As mãos apoiam a nuca ou flexionam-se os cotovelos sobre o peito (braços em formato de $\mathrm{x}$ ). 0 avaliador segura os pés do avaliado. 0 movimento deve ser completo, até os cotovelos encostarem-se às coxas. Somente serão validadas as repetições que forem completas. 
Teste de flexão de braços (Canadian Standardized Test of Fitness), esse teste consiste na exaustão do indivíduo em executar o maior número de repetições. A posição inicial do avaliado varia de homem para mulher. Para o indivíduo do sexo feminino, lhe é permitido encostar os joelhos no solo. Os cotovelos devem estender-se completamente e na volta para a posição inicial flexiona-se até próximo ao solo (cotovelo na posição de $90^{\circ}$ ). Ao indivíduo do sexo masculino, não the é permitido encostar os joelhos no solo. Os joelhos devem-se manter completamente estendidos, enquanto os cotovelos estendem-se totalmente e na volta para a posição inicial flexionam-se até próximo ao solo (cotovelo $90^{\circ}$ ). Somente serão válidas as posições executadas de maneira correta (Protocolo do CSTF) 9 .

Teste de impulsão horizontal ${ }^{10}$, este teste tem como objetivo medir a potencia de membros inferiores. 0 material necessário é uma fita métrica fixada no chão, uma régua ou fita métrica para marcar o ponto de chegada e papel para anotação. 0 teste consiste em três tentativas. É permitido o movimento de braços e tronco. 0 avaliado deve saltar ao sinal do avaliador, e é registrada a marca (calcâneo) do pé que estiver mais próximo do ponto de partida; vale o melhor dos três saltos executado pelo avaliado. Os mesmos procedimentos deste teste podem ser aplicados em crianças.

COLETA DE DADOS

Para que isso acontecesse, foi necessário o agendamento de reuniões com os pais dos indivíduos, no ato de haver o consentimento dos mesmos nos testes aplicados. Tais testes foram aplicados no ginásio da Faculdade de Educação Física e Fisioterapia (FEFF). Os indivíduos foram avaliados de forma individual. Os testes aplicados foram executados na seguinte forma seqüencial para todos os indivíduos avaliados: primeiramente foi feito 0 teste de resistência abdominal de 1 minuto, depois o teste de flexão de braços e por último o teste de impulsão horizontal.

\section{RESULTADOS}

Os indivíduos que participaram dos testes de aptidão física encontravam-se com diferentes faixas etárias, onde 3 (três) dos alunos apresentaram está com idade entre 17 a 28 anos e os outros 3 (três) alunos apresentaram ter idade entre 29 a 36 anos, conforme o quadro 1.
QUADRO 1 - Faixa etária dos indivíduos

\begin{tabular}{cc}
\hline IDADE & QUANTIDADE DE INDIVÍDUOS \\
\hline 17 A 28 ANOS & 3 \\
\hline 29 A 36 ANOS & 3 \\
\hline TOTAL & 6
\end{tabular}

FONTE: Programa de Atividades Motoras para Deficiente

PATOLOGIA

Os indivíduos que participaram da pesquisa possuíam diferentes tipos de patologias, dentre elas, 1 (um) dos alunos apresentava ter esquizofrenia, 2 (dois) dos alunos avaliados apresentaram ter paralisia cerebral, 1 (um) dos alunos apresentou ter transtorno do desenvolvimento e os outros 2 (dois) alunos apresentaram ter retardo mental, como mostra o quadro 2.

QUADRO 2 - Patologia dos indivíduos.

\begin{tabular}{cc}
\hline PATOLOGIA & QUANTIDADE DE INDIVÍDUOS \\
\hline Esquizofrenia & 1 \\
\hline Paralisia Cerebral & 2 \\
\hline Transtorno do Desenvolvimento & 1 \\
\hline Retardo Mental & 2 \\
\hline TOTAL & 6 \\
\hline
\end{tabular}

FONTE: Programa de Atividades Motoras para Deficiente

TESTE DE FLEXÃO DE BRAÇOS (MASCULINO E FEMININO)

Para mensurar a resistência de membros superiores dos indivíduos do sexo masculino e feminino, foi utilizado o teste de flexão de braços do (Canadian Standardized Test of Fitness), onde os avaliados foram classificados de acordo com o padrão do protocolo de avaliação do CSTF. 0 nível alcançado pelos indivíduos na tabela de classificação é referente às flexões de braços com o maior número de repetições que os avaliados puderam executar até a exaustão dos mesmos, como mostra os quadros 3 e 4 . 


\begin{tabular}{cc}
\hline HOMENS & QUANTIDADE DE INDIVÍDUOS \\
\hline EXCELENTE & 0 \\
\hline MÉDIA + & 0 \\
\hline MÉDIO & 0 \\
\hline MÉDIA - & 1 \\
\hline FRACO & 2 \\
\hline TOTAL & 3 \\
\hline
\end{tabular}

QUADRO 4 - Classificação dos indivíduos do sexo feminino no teste de flexão de braços.

\begin{tabular}{cc}
\hline MULHERES & QUANTIDADE DE INDIVÍDUOS \\
\hline EXCELENTE & 0 \\
\hline MÉDIA + & 0 \\
\hline MÉDIO & 0 \\
\hline MEDIA - & 1 \\
\hline FRACO & 2 \\
\hline TOTAL & 3 \\
\hline
\end{tabular}

TESTE DE RESISTÊNCIA ABDOMINAL DE 1 MINUTO (MASCULINO E FEMININO)

Para mensurar a resistência dos músculos abdominais e os flexores do quadril dos indivíduos, foi utilizado o teste de resistência abdominal de 1 minuto, onde indivíduos avaliados executaram o maior número de repetições no tempo determinado de 1 minuto, de forma que os mesmo foram classificados conforme o padrão do protocolo de avaliação da Young Men's Christian Association (YMCA). Os níveis alcançados pelos indivíduos no quadro de classificação podem ser visualizados nos quadros 5 e 6. teste de resistência abdominal de 1 minuto (YMCA)

\begin{tabular}{|c|c|}
\hline MASCULINO & QUANTIDADE DE INDIVÍDUOS \\
\hline EXCELENTE & 0 \\
\hline BOM & 0 \\
\hline MÉDIA + & 0 \\
\hline MÉDIO & 0 \\
\hline MÉDIA - & 0 \\
\hline FRACO & 0 \\
\hline MUITOFRACO & 3 \\
\hline TOTAL & 3 \\
\hline
\end{tabular}

QUADRO 6 - Classificação dos indivíduos do sexo feminin no teste de resistência abdominal de 1 minuto (YMCA)

\begin{tabular}{cc}
\hline FEMININO & QUANTIDADE DE INDIVIDUO \\
\hline EXCELENTE & 0 \\
\hline BOM & 0 \\
\hline MÉDIA + & 0 \\
\hline MÉDIO & 0 \\
\hline MÉDIA - & 1 \\
\hline FRACO & 0 \\
\hline MUITO FRACO & 2 \\
\hline TOTAL & 3 \\
\hline
\end{tabular}


TESTE DE IMPULSÃO HORIZONTAL (JOHNSON E NELSON, 1979), MASCULINO E FEMININO

Para medir a potência de membros inferiores dos indivíduos avaliados, foi utilizado o teste de impulsão horizontal, onde cada um dos indivíduos teve três oportunidades para saltar o mais longe possível. Dessa forma os indivíduos foram classificados de acordo com o padrão do protocolo de avaliação de Johnson e Nelson ${ }^{10}$, como mostram os quadros 7 e 8.

QUADRO 7 - Classificacãão dos indivíduos do sexo masculino

no teste de impulsão horizontal

\begin{tabular}{cc}
\hline MASCULINO & QUANTIDADES DE INDIVÍDUOS \\
\hline FRACO & 3 \\
\hline REGULAR & 0 \\
\hline BOM & 0 \\
\hline MUITO BOM & 0 \\
\hline EXCELENTE & 0 \\
\hline TOTAL & 3 \\
\hline
\end{tabular}

QUADRO 8 - Classificação dos indivíduos do sexo feminino

no teste de impulsão horizontal

\begin{tabular}{cc}
\hline FEMININO & QUANTIDADE DE INDIVÍDUOS \\
\hline FRACO & 3 \\
\hline REGULAR & 0 \\
\hline BOM & 0 \\
\hline MUITO BOM & 0 \\
\hline EXCELENTE & 0 \\
\hline TOTAL & 3 \\
\hline
\end{tabular}

\section{DISCUSSÃo}

Após os resultados dos testes de aptidão física dos indivíduos com deficiência intelectual, foi possível observar que os mesmos mantiveram-se com baixos níveis nas classificações, porém é de grande importância destacar esses resultados, pois tais indivíduos podem contribuir para implementação de programas de educação física.

A avaliação da aptidão física voltada à saúde é de suma importância para a regulamentação de programas de educação física tanta para indivíduos com deficiência como para indivíduos sem deficiência, baseando-se nas especificidades e dificuldades individuais ${ }^{11}$.

Assim, os indivíduos deficientes intelectuais avaliados nos teste de aptidão física com baixos índices nos parâmetros estabelecidos nos quadros de classificação, podem ter oportunidade para participarem de atividades físicas que valorizem o potencial e as suas capacidades através de programas que se baseiam nas necessidades e cuidados dos indivíduos.

Dessa forma, devemos avaliar de forma minuciosa o indivíduo e o suporte que ele precisa. Deve ser feita uma avaliação detalhada do indivíduo e o apoio que ele necessita o que irá permitir analisar de forma separada as áreas em que podem existir necessidades, sendo providenciada a intervenção que reconheça a relação existente dos aspectos considerados ${ }^{12}$.

Os estudos relacionados à aptidão física de maneira geral possibilitam a identificação de características inerentes a algumas e as diferenças existentes entre elas e outras populações, além de permitir a comparação de subgrupos de uma mesma região ${ }^{13}$. 0 autor afirma que o estudo de variações intra e inter-populações poderão enriquecer os conhecimentos sobre os fatores e as relações existentes na determinada população ou subgrupo, podendo assim fazer comparações entre elas.

Os estudos feitos de populações especiais como das pessoas com deficiência intelectual buscam contribuir no conhecimento acerca dessa determinada deficiência, procurando proporcionar atividades alternativas que possam estimular o potencial e as capacidades desta população ${ }^{11}$.

Existem várias razões para avaliar pessoas com deficiência. Os dados obtidos da avaliação podem ser usados para nortear os indivíduos para programas que aperfeiçoem o aprendizado. Dessa maneira, a avaliação pode identificar pontos fracos e fortes do indivíduo e determinar se o mesmo necessita de uma avaliação mais aprofundada. Além de que, a avaliação pode guiar o desenvolvimento de objetivos e metas de um programa de atividade física, bem como auxiliar na construção de relações, estimulando assim a comunicação entre pais e responsáveis e profissionais da área de educação física ${ }^{14}$. 
Percebe-se que os testes de aptidão física tem diversos objetivos e direcionamentos diferentes para determinadas populações, grupos e subgrupos. Os testes aplicados nos indivíduos da pesquisa tinham como objetivo verificar as potencialidades e capacidades do mesmo, no intuito de oferecer melhores condições na qualidade de vida e principalmente alertar sobre os riscos de doenças desse grupo de pessoas.

Os objetivos do teste de aptidão física são: a) Educar os indivíduos sobre a sua situação atual de aptidão relativa aos padrões relacionados à saúde; b) Apresentar dados que auxiliem o desenvolvimento da prescrição de exercícios para todos os componentes de aptidão; c) Criar uma base de dados que possa ser ampliada e que possa mostrar a avaliação do progresso obtido pelos indivíduos através do programa de exercício; d) estimular os indivíduos através dos estabelecimentos de metas razoáveis e alcançáveis de boa aptidão física; e) Reduzir os riscos de doenças ${ }^{15}$.

Os estudos realizados na classe da aptidão física mostram bastante a preocupação e o interesse em melhor compreender a relação com a saúde e o bem estar dos jovens deficientes, assim como a associação estreita com o desempenho desportivo e motor.

De uma cuidada revisão da literatura, verifica-se que os parâmetros inferiores de aptidão física obtidos por indivíduos com Deficiência Mental, mostram pouco interesse em estudar adultos classificados como deficientes mentais. Esta situação parece estar associada com os déficits mentais observados neste grupo, o que poderá, de fato, desencorajar muitos profissionais a trabalhar com esta população. Ainda mais, que na área da deficiência mental são poucas as informações sobre de que forma estes indivíduos reagem ao exercício ${ }^{16}$.

É percebível que os testes de aptidão física são ferramentas de extrema importância para avaliar indivíduos com ou sem deficiência, também é possível observar que os testes são direcionados para diversos objetivos e razões diferentes dos estudos feitos. Porem há uma escassez de estudos na área de aptidão física de pessoas com deficiência mental, em particular os adultos, o que nos leva a acreditar que existe pouco interesse em estudar indivíduos que são identificados com deficiência mental, uma vez que entendemos que os testes de aptidão física são de grande importância para esse grupo de indivíduos, pois os testes podem alertar os indivíduos para os riscos de doenças e para que se mantenham com uma boa qualidade de vida.

\section{REFERÊNCIA}

1. Araújo DSMS; Araújo CGS (2000). Aptidão física, saúde e qualidade de vida relacionada à saúde en adultos. Revista Brasileira de Medicina do Esporte 6(5):194-203.

2. Gallahue D; Donnely FC (2008). Educação física desenvolvimentista para todas as crianças. Phorte. 3. Bohme MTS (2003). Relações entre aptidão física, esporte etreinamento esportivo; Relation among physical fitness, sport and sport training. Rev. bras. ciênc. mov, 11(3):97-104

4. Guedes DP: Guedes JERP: Barbosa DS; Oliveira JA (2002). Atividade física habitual e aptidäo física relacionada à saúde em adolescentes; Habitual physical activity and health-related physical fitness in adolescents. Rev. bras. ciênc. mov, 10(1):13-21.

5. Amiralian MLT; Elizabeth BP; Maria IG; Ida L; Elcie FSM: Luiz P (2000). Conceituando deficiência Rev. Saúde Pública, 34 (1): 97-103.

6. Gil AC (2002). Como classificar as pesquisas. Como elaborar projetos de pesquisa. (4): 41-56.

7. Nascimento ACS (2000). Pedagogia do esporte 0 atletismo: considerações acerca da iniciação e da especialização esportiva precoce. Campinas-SP: (Dissertação Mestrado)-FEF/UNICAMP.

8. Fontura A; Formentin C; Abech E (2008). Guia Pràtico de Avaliação Física. Uma abordagem didática, abrangente e atualizada. Phorte Editora, Brasil.

9. Morrow J; Jackson A; Disch J; Mood D (1995). Measurement and evolution in human performance. Human Kinectics, printed in the united states of America, Illionis. 10. Johnson BL; Nelson JK (1979). Pratical measurement for evolution in physical education 4ed. Edina, MN: Burguerss Publishing

11. Bacciotti SM (2009). Avaliação da aptidão física relacionada à saúde em indivíduos de 8 a 17 anos com deficiência mental da APAE de Campo Grande-MS. Dissertação apresentada como requisito parcial para obtenção de título de Mestre no Programa de Pós-Graduação Stricto Sensu da Faculdade de Ciências da Saúde, da Universidade de Brasília.
12. Cunha C; Brito J (2004). Programa de Animação Esportiva em Adultos Portadores de Deficiência Mental - aspectos metodológicos e operacionais. II Congresso Internacional da Montanha, Portugal.

13. Guedes DP (1999). Educação para a saúde mediante programas de Educação Física escolar. Revista Motriz. Rio Claro, 5(1).

14. Combs S (2003). Avaliação para populações com Necessidades Especiais IN TRISCHLER, K. Medidas e avaliação em educação física e esporte de barrow e McGee. Barurei, SP; Manole.

15. Hobold E (2003). Indicadores de Aptidão Física Relacionada à Saúde de Crianças e Adolescentes no Município de Marechal Cândido Rondon - Paranà Brasil. Dissertação Apresentada ao Centro de Desportos da Universidade Federal de Santa Catarina Como Requisito Parcial à Obtenção do Título de Mestre em Educação Física na Área de Concentração de Atividade Física Relacionada à Saúde. Fevereiro.

16. Maia LPR (2002). Estudo dos níveis de aptidão física em indivíduos deficientes mentais com e sem síndrome de Down. Dissertação apresentada às provas de Mestrado em Ciência do Desporto na Área de Especialização em Atividade Física Adaptada. Faculdade de Ciências do Desporto e de Educação Física - Universidade do Porto. Porto. 